- Original Article

\title{
Perceived Stress, Alexithymia, and Psychological Health as Predictors of Sedative Abuse
}

\author{
Nader Rajabi Gilan', Ali Zakiei',*, Sohyla Reshadat', Saeid Komasi², Seyed Ramin Ghasemi' \\ 'Social Development and Health Promotion Research Center, Kermanshah University of Medical Sciences, Kermanshah, Iran \\ ${ }^{2}$ Cardiac Rehabilitation Center, Imam Ali Hospital, Kermanshah University of Medical Sciences. Kermanshah, Iran
}

Background: The harmful effects of sedative medications and substances in conjunction with limited research regarding predictive psychological constructs of drug abuse necessitate further investigation of associated factors. Therefore, the present study aimed to elucidate the roles of perceived stress, alexithymia, and psychological health as predictors of sedative abuse in medical students.

Methods: In this cross-sectional study, 548 students at Kermanshah University of Medical Sciences, Iran, were selected using stratified random sampling. The data were obtained using the Perceived Stress Scale, an alexithymia scale (Farsi version of the Toronto Alexithymia Scale-20), and a General Health Questionnaire to assess psychological health. Data were analyzed using discriminant analyses.

Results: The results demonstrated that the user and non-user of sedative substances groups had significantly different predictive variables (except for social function disorder) $(\mathrm{P}>0.05)$. Physical complaints, alexithymia, and perceived stress, which had standard coefficients of $0.80,0.60$, and -0.27 , respectively, predicted sedative drug use.

Conclusion: The results of the present study indicate that perceived stress, alexithymia, physical complaints, anxiety, and depression are associated with sedative drug abuse.

Keywords: Perceived Stress; Affective Symptoms; Psychological Disorders; Sedative Drug Abuse; University Students 


\section{INTRODUCTION}

Sedative substances suppress the central nervous system, decrease anxiety, and promote relaxation and sleep. Additionally, some sedatives cause amnesia, muscle relaxation, or have anticonvulsive effects. Common sedative/hypnotic substances include alcohol, barbiturates, and chloral hydrate, ${ }^{1)}$ and these drugs are frequently used to treat sleep and anxiety disorders. ${ }^{2)}$ Sedatives may result in reduced concentration, as well as impaired memory and motion coordination. They may also produce effects such as hangover, slurred speech, uncoordinated motion, gait instability, somnolence, dry mouth (xerostomia), and unstable mood. ${ }^{1,3)}$ Illegal use and abuse of sedative substances contribute to physical and psychological damage in those who use them. These drugs disrupt normal physiological activity and may lead to death when overused. Dependence on sedative drugs is also problematic from a sociological perspective, as it may damage other aspects of the user's life, including family, work, and finances. ${ }^{4)}$ Despite these consequences of sedative drug use, demographic and clinical factors related to sedative drug abuse have not been elucidated. ${ }^{5,6)}$

Current concerns regarding sedative drug abuse can be categorized into 2 broad yet distinct problems. Firstly, overuse occurs by patients for whom the medications are prescribed. Secondly, there are people who abuse the drugs by taking them in ways that work against the prescription objective. ${ }^{7)}$ There are harmful impacts of illegal sedative drug use, especially among university students, so factors associated with of their use should be elucidated. Various factors, including cultural, social, and psychological contributors, as well as personality traits may be important contributors. We hypothesized that perceived stress might be related to sedative drug use. Perceived stress is a psychological state or process through which individuals perceive threat to their physical and psychological well-being. According to this definition, stress depends on the perception and understanding of the individual, so a situation may be perceived as harmless to one individual yet threatening to another. ${ }^{8)}$

Conversely, previous research has demonstrated that disorders such as antisocial personality disorder, some phobia and anxiety disorders, major depressive disorder, and depressed mood have the strongest relationships with drug abuse and dependence. ${ }^{7,9)}$ However, the role of psychological disorders specifically in sedative drug use has not been characterized, so this characterization is one of the objectives of the present study. Previous studies have suggested contributions of personality factors in drug abuse; ${ }^{7,10,11)}$ therefore we evaluated the relationship between alexithymia and sedative drug use.

The term 'alexithymia' was developed by psychotherapist Peter Sifneos in 1973, and is a personality construct characterized by the inability to cognitively process emotional information or to regulate emotions. ${ }^{12)}$ Those with this impaired emotional ability are unable to identify and distinguish their emotions, and cannot understand and describe their thoughts and feelings. ${ }^{13)}$ People with alexithymia exaggerate physical excitement and misinterpret somatic symptoms of emotional arousal. It is believed that impaired emotional ability is a risk factor for many psychological disorders, because individuals with this condition are distressed by experiencing emotions that they cannot express verbally. This dysfunction hinders emotional regulation and impedes adjustment to various circumstances or events. One hypothesis is therefore that individuals with alexithymia engage in harmful non-verbal actions as a substitute to expressing their emotions verbally. Behaviors such as tearing, destruction, and breaking things, drinking alcohol, and drug use are examples of these harmful non-verbal activities. ${ }^{14)}$ Some authors have emphasized that alexithymia is related to drug abuse, ${ }^{15)}$ such as by reporting findings that $45 \%$ to $67 \%$ of alcohol dependent individuals have been identified as alexithymic. ${ }^{16-18)}$

Previous research indicates that the prevalence of psychological disorders is higher in drug-dependent individuals than in non-dependent persons, ${ }^{19)}$ and that the psychological health of patients with psychedelic dependency is lower than in non-dependent individuals. ${ }^{20)}$ However, other studies have demonstrated that there is no relationship between anxiety and drug abuse. ${ }^{4)}$ The present study aimed to resolve inconsistencies in the literature and expand this field of research by investigating whether the linear combination of perceived stress, alexithymia, and psychological disorders predict sedative drug abuse.

\section{METHODS}

\section{Study Design}

This cross-sectional study examined the demographics and clinical state of students attending Kermanshah University of Medical Sciences, in Kermanshah, Iran during the 2013-2014 academic year. This university is a state school in Western Iran, which offers medical majors such as medicine, pharmacy, public health, nursing, and paramedics to students from all areas of Iran. The study was registered with the Kermanshah University of Medical Sciences Research Center (with the registration number of 93,195) and received an ethical license from the university ethics committee.

\section{Inclusion Criteria}

The inclusion criteria for this study were: (1) 18 to 35 years of age; (2) no history of psychotic disorder; and (3) interest in study participation.

\section{Participants and Procedure}

The sample was composed of 600 students from different faculties, who were selected using stratified random sampling according to the Cochran formula. Forty students were excluded 
from the study because they did not meet inclusion criteria. Next, the remaining 560 students were interviewed by a clinical psychologist and were provided with required explanations to answer the questionnaire and gain written informed consent for participation in the study. After questionnaire completion, 12 participants were excluded due to having altered or incomplete information. Therefore, data from 548 participants was included in the final analyses. Participants were classified as sedative drug users $(n=250)$ or non-users $(n=298)$. The user group was selected based on the international treaties of drug control criteria. In the international treaties of drug control, drug consumption is defined as abuse regardless of the amount and frequency of consumption. ${ }^{1)}$ In order to verify participants' health status, they presented their health certificate (health record) to the research team.

\section{Instruments}

The research questionnaires had 4 components. The first component collected demographic information including age, gender, and consumption or non-consumption of sedative drugs, including the amount of consumption. The 28-item General Health Questionnaire (GHQ-28), Farsi version of the Toronto Alexithymia Scale-20 (FTAS-20), and the Perceived Stress Scale (PSS) were also completed.

\section{1) General Health Questionnaire}

The 28-item Goldberg Scale test (1998) ${ }^{21)}$ was used to assess general health of the participants. The reliability coefficients of the subscales of this questionnaire have been reported as 0.92 , $0.88,0.91$, and 0.83 , using Cronbach's alpha. The reliability coefficient was estimated at 0.84 using the Spearman-Brown method and the internal consistency coefficients were estimated at $0.79,0.91$, and $0.83 .{ }^{21)}$ GHQ-28 is a commonly used 28item questionnaire specially designed to detect diverse psychiatric symptoms. The GHQ has been translated into more than 30 languages, including Persian, due to its use as a powerful screening instrument. In an Iranian national study, the GHQ was validated in a sample of 35,014 Iranians. ${ }^{22)}$ The GHQ is a self-administered questionnaire that effectively detects minor, non-psychotic psychiatric disorders in general practice. It has previously been used with dermatologic patients and determined to be a valid and reliable instrument. ${ }^{23)}$ The Cronbach's alpha for that study was 0.91 .

\section{2) Toronto Alexithymia Scale-20}

Alexithymia was assessed by the 20 -item Toronto Alexithymia Scale (TAS-20), in order to assess the severity of alexithymia. ${ }^{24,25)}$ The 20-item self-report instrument uses a 5-point Likert-type scale ranging from 1 (strongly disagree) to 5 (strongly agree). Total scores therefore range from 20 to 100, with higher scores indicating higher levels of alexithymia. The TAS-20 assesses 3 factors: difficulty identifying feelings (DIF); difficulty describing feelings (DDF); and externally oriented cognitive style of thinking (EOT). The psychometric features of the FTAS-20 have been confirmed by numerous studies. In the Farsi version of the FTAS20, Cronbach's alpha coefficients for general alexithymia and its subscales of DIF, DDF, and EOT were calculated as 0.85, 0.82, 0.75 , and 0.72 , respectively, which indicates high internal validity of the scale. The test-retest reliability of the FTAS-20 with a sample of 67 participants tested 4 weeks apart was good, with $r=0.80$ to $r=0.87$ for general alexithymia and the different subscales. The concurrent validity of the FTAS-20 was evaluated and confirmed using correlation between subscales and emotional intelligence, psychological well-being, and psychological frustration. Pearson correlation coefficients indicated that there is a significant correlation between scores on the general alexithymia scale and emotional intelligence, psychological wellbeing, and psychological frustration. The correlation coefficients between the alexithymia subscales and these variables were also significant. The results of confirmatory factor analysis have also supported factors such as DIF, DDF, and EOT on the Farsi version of the FTAS-20. ${ }^{26)}$ Ghorbani et al. ${ }^{27)}$ estimated the validity of the scale among Iranian and American samples and calculated coefficients for subscales including DIF, DDF, and EOT as $0.50,0.74$, and 0.61 , respectively, for the Iranian samples. For the American group, the coefficients were 0.60, 0.82, and 0.77, respectively. In the present study, Cronbach's alpha was 0.76 for the FTAS-20.

\section{3) The Perceived Stress Scale}

This scale, which was developed by Ghorbani et al. in 2002, assesses perceived typical stress over the past month. The scale is designed for use with participants who have achieved a university degree. Ghorbani et al. reported Cronbach's alpha at 0.84 and 0.86 for this scale. In a study done by Ghorbani et al., ${ }^{27)}$ Cronbach's alpha in an American sample was 0.86 and in an Iranian sample it was calculated as 0.81 . In the present study, the Cronbach's alpha was 0.91 .

\section{Statistical Analyses}

Data from 548 participants were analyzed with binary analyses, using IBM SPSS software ver. 21.0 (IBM Co., Armonk, NY, USA), in order to predict whether students were in the user or nonuser groups. First, the definite binary analysis was determined based on the following variables: perceived stress, alexithymia, and psychological disorders (physical complaints, anxiety, lack of sleep, social function disorder, and depression). In order to use binary analyses as our statistical method, the assumptions of this statistical method (M-box test, multiple linear regression, and equality of means for predicting variables) were assessed, with results confirming the appropriateness of this method. 


\section{RESULTS}

There were 106 men and 144 women in the user group, with mean age of $22.10 \pm 2.39$ years, and there were 100 men and 198 women in the non-user group, with mean age of $22.10 \pm 1.45$ years. Table 1 shows means and standard deviations for predicting variables by group. As shown in Table 1, the means for the user group are higher for all variables compared to the non-user group. In addition, significant differences were found between groups for all variables, except for social function disorder.

The results in Table 1 indicate that the mean alexithymia score in the sedative drug user group was 51.73, compared to 47.38 in the non-user group. The mean perceived stress score for the user group was 41.08 , and in the non-user group it was 39.69. Scores on the physical complaints subscale were 7.16 in the user group and 5.32 in the non-user group. The anxiety subscale score for the user group was 7.0, and in the non-user group it was 5.33. Finally, on the depression subscale, the user group score was 4.93 and the non-user group score was 3.52.

Binary analysis yielded a Wilks' Lambda value $>1$ (0.91), which

Table 1. The characteristics of sedative users and non-users

\begin{tabular}{llcccc}
\hline Variable & Group & Mean \pm SD & $\begin{array}{c}\text { Wilks' } \\
\text { Lambda }\end{array}$ & F & P-value \\
\hline Alexithymia & User & $51.73 \pm 10.4$ & 0.95 & 25.46 & $0.001^{*}$ \\
& Non-user & $47.38 \pm 10.80$ & & & \\
Perceived stress & User & $41.08 \pm 7.17$ & 0.99 & 5.22 & $0.02^{* *}$ \\
& Non-user & $39.69 \pm 7.05$ & & & \\
Physical complaints & User & $7.16 \pm 3.71$ & 0.93 & 39.52 & $0.001^{*}$ \\
& Non-user & $5.32 \pm 3.10$ & & & \\
Anxiety & User & $7.00 \pm 4.77$ & 0.96 & 19.54 & $0.001^{*}$ \\
& Non-user & $5.33 \pm 4.05$ & & & \\
Social function disorder & User & $10.89 \pm 3.30$ & 0.99 & 0.14 & 0.70 \\
& Non-user & $10.77 \pm 3.51$ & & & \\
Depression & User & $4.93 \pm 5.33$ & 0.98 & 12.26 & $0.001^{*}$ \\
& Non-user & $3.52 \pm 4.09$ & & & \\
\hline
\end{tabular}

${ }^{*} \mathrm{P}<0.01 .{ }^{* *} \mathrm{P}<0.05$.

Table 2. A summary of information related to the discriminant function of variables

\begin{tabular}{cccccccc}
$\begin{array}{c}\text { No. of } \\
\text { functions }\end{array}$ & Value & $\begin{array}{c}\% \text { of } \\
\text { Variance }\end{array}$ & $\begin{array}{c}\text { Canonical } \\
\text { correlation }\end{array}$ & $\begin{array}{c}\text { Wilks' } \\
\text { lambda }\end{array}$ & Chi-square & df & P-value \\
\hline 1 & 0.10 & 100 & 0.30 & 0.91 & 50.41 & 6 & $0.001^{*}$ \\
\hline
\end{tabular}

${ }^{*} \mathrm{P}<0.01$. was statistically significant $(\mathrm{P}>0.001)$. The binary function for the combination of research variables is significant. Given that the special (0.10) and the high chi-square amounts (50.41), the obtained binary function has a suitable discriminatory power for the description of the dependent variables variance which is effective in grouping (in the two group levels). In addition, the normal correlation (0.30) and Wilks' Lambda value >1 (0.91) demonstrate that nearly 91 percent of the variance between groups is described by 5 predicting variables (all variables assessed in the current study except for social function disorder). A summary of information related to the discriminant function of the variables is shown in Table 2.

In order to determine the strongest predicting variables for sedative drug use, binary analysis was conducted. The results of the analysis indicate that physical complaints $(0.80)$ were the strongest predictor of sedative drug use. Alexithymia and perceived stress were the next strongest predictors, with values of 0.60 and -0.27 respectively. Anxiety was the weakest predictor among predictors that were statistically significant, as indicated in Table 3.

Standard and non-standard coefficients are indices of the portion of variance accounted for, which is interpreted as the relative importance of the predicting variables, and are used to create predictive equations. Using non-standard coefficients obtained by the present study, the binary function equation could be written as follows: $\mathrm{D}=-2.80+0.06\left(\mathrm{X}_{1}\right)-0.04\left(\mathrm{X}_{2}\right)+$ $0.23\left(\mathrm{X}_{3}\right)+0.001\left(\mathrm{X}_{4}\right)-0.009\left(\mathrm{X}_{5}\right)$.

Inserting the scores of each participant into the equation above, a binary score would be obtained and could be used to predict an individual's group membership. The mean calculations for the user and non-user groups are 0.34 and -0.28 , respectively. Positive values indicate membership in the user group and negative scores indicate membership in the non-user group, with an average of 0 when all participants are included.

\section{DISCUSSION}

The present study was conducted in order to investigate the contributions of perceived stress, alexithymia, and psychological health in predicting sedative abuse amongst students at Kermanshah University of Medical Sciences. The results of the

Table 3. Standard, non-standard, and structural coefficients of the discriminant function variables

\begin{tabular}{|c|c|c|c|c|c|c|c|c|c|c|}
\hline \multirow{2}{*}{ Predictor variable } & \multicolumn{3}{|c|}{ Standard coefficients } & \multicolumn{3}{|c|}{ Non-standard coefficients } & \multicolumn{3}{|c|}{ Structural coefficients } & \multirow{2}{*}{ Code } \\
\hline & Male & Female & Total & Male & Female & Total & Male & Female & Total & \\
\hline Alexithymia & 0.17 & 0.80 & 0.60 & 0.02 & 0.07 & 0.06 & 0.92 & 0.73 & 0.69 & $x_{1}$ \\
\hline Perceived stress & 0.12 & -0.46 & -0.27 & -3.66 & -2.07 & -0.04 & 0.30 & 0.16 & 0.31 & $x_{2}$ \\
\hline Physical complaints & 1.02 & 0.74 & 0.80 & 0.33 & 0.21 & 0.23 & 0.63 & 0.72 & 0.86 & $X_{3}$ \\
\hline Anxiety & 0.15 & -0.31 & 0.006 & 0.03 & -0.07 & 0.001 & 0.48 & 0.47 & 0.61 & $X_{4}$ \\
\hline Depression & -0.53 & 0.22 & -0.04 & -0.11 & -0.06 & -0.009 & 0.37 & 0.47 & 0.48 & $X_{5}$ \\
\hline Constant & \multicolumn{10}{|c|}{-2.80} \\
\hline
\end{tabular}


study demonstrated that perceived stress, alexithymia, physical complaints, anxiety, and depression predict sedative drug use. However, social function disorder does not predict sedative drug use. Of the predicting variables, physical complaints were the strongest predictor of sedative drug use. We hypothesize that regardless of the initial cause of the physical symptoms, individuals may use drugs to alleviate the associated pain.

There have not been previous studies investigating the contributions of perceived stress, alexithymia, physical complaints, and depression as predictors of sedative drug use, so comparison with the literature is not possible. However, our findings regarding the relationship between anxiety and sedative drug use are inconsistent with a previous study ${ }^{4}$ because the present findings indicate that anxiety is a stronger predictor of sedative drugs use than was demonstrated by the previous study. However, the literature consistently indicates that a relationship exists. $^{15,16)}$

The present results also demonstrate that alexithymia predicts sedative drug use, which may result from people with alexithymia having tension resulting from experiencing the physical aspects of emotions that are not verbally expressed. ${ }^{13)}$ The inability to express emotion may hinder emotional regulation and prohibit successful adjustment, leading to these individuals having reduced adjustment capabilities in stressful situations. People who can express their emotions are released from mental tension, but people with alexithymia are unable to share their feelings with others. It can therefore be argued that alexithymia is a cognitive emotional trait and those who have it are unable to regulate and understand their own emotional arousals. When emotional information is not understood and assessed through cognitive processes, people will experience distress, thereby disrupting their emotions and recognition systems. These people are usually unable to recognize, understand, or describe their emotions due to a lack of emotional awareness, and have little ability in coping with stressful conditions. One strategy to control stress, especially with regard to negative emotions, is to drain and express the emotions arising from tension, which is exactly what people with alexithymia are unable to do. We hypothesize that those with alexithymia therefore tend to use drugs and sedative materials to minimize their tension, essentially substituting drugs for emotional expression. Previous studies have indicated that people with alexithymia are more likely to use drugs and drink alcohol, compared to those without alexithymia. ${ }^{14)}$

The results of the present study also demonstrate that perceived stress predicts sedative drug use, possibly because stress and tension produce restlessness and the individual therefore uses drugs to reduce the tension. In this situation, the drugs are a reaction to stress, which is a negative emotion oriented reaction approach. The participant population for the present study were students, a group that typically experiences particular types of stress, and their educational experience may contribute to sedative drug use. In order to explain the relationship between anxiety and stress as predictors of sedative drug use, one possibility is that consumption of sedative drugs may minimize anxiety and the associated symptoms. ${ }^{2)}$ Alternately, those with depression may use sedative drugs to forget and suppress their disappointments and minimize their depression. The primary symptoms of anxiety are anguish and worry, which may disrupt individual's lives to a serious enough degree that they use sedative drugs as a coping mechanism. Moreover, due to the general belief that sedative drugs are tranquilizers, and therefore soothe anxiety and stress, these drugs may be appealing to many people.

Previous research findings suggest that for men, physical complaints best predicted sedative drug use, compared to alexithymia for women. Accordingly, in the present study alexithymia predicted sedative drug use. This finding may be due to cultural factors because in Middle Eastern culture, women are discouraged from expressing emotions. Culture may also be an important factor in the relationship between sedative abuse in men and physical complaints.

The most reliable studies on personality in patients who are dependent on sedative drugs indicate that these people are not diagnosed with psychopathology. A common assumption is that dependence on these drugs is a side effect of mental disorders, and leads to various complications in each of the mental disorders. Based on existing studies, many substance abusers who seek treatment are dually diagnosed, meaning that in addition to the addiction, they have other syndromes such as depression, a personality disorder, or a combination of both.

Conversely, various surveys have found disorders associated with sedative abuse, including affective disorders, ${ }^{28,29)}$ personality disorders, ${ }^{30,31)}$ and psychotic disorders. ${ }^{28)}$ Of the affective disorders (depression and anxiety), personality disorders (antisocial and borderline personality disorders), and psychotic disorders, schizophrenia has been the disorder most strongly associated with sedative abuse.

The present study relied on self-report, which is a limitation, so other methods should be used in future studies evaluating sedative abuse. Furthermore, there are many conditions that are comorbid with sedative use, so future studies should attempt to control for these potentially confounding variables.

In conclusion, perceived stress, alexithymia, physical complaints, anxiety, and depression are strong predictors of sedative drug use. Given that the present study investigated sedative use in university students, caution is warranted with extending the results to other social groups. Therefore studying other groups is recommended as a goal of future studies. Future studies should also focus on the role of personality and psychological factors as predictors of drug and alcohol abuse. Further understanding of the underlying causes and factors contributing to sedative drug 
abuse will lead to meaningful preventative strategies, and treatments. The present study sought to contribute to these objectives. The present findings suggest that the contributions of perceived stress, alexithymia, and psychological disorders should be considered in developing preventative strategies for reducing sedative drug abuse.

\section{CONFLICT OF INTEREST}

No potential conflict of interest relevant to this article was reported.

\section{REFERENCES}

1. Shafiei S, Jafari S, Raeisi H, Nikfarjam A. Glossary of demand reduction. Tehran: Sepidbarg Pub; 2011.

2. O'Brien CP. Benzodiazepine use, abuse, and dependence. J Clin Psychiatry 2005;66 Suppl 2:28-33.

3. Buffett-Jerrott SE, Stewart SH. Cognitive and sedative effects of benzodiazepine use. Curr Pharm Des 2002;8:45-58.

4. Haghighat M, Tabatabaee SM, Boogar IR. Relation of anxiety, sleep disorders and drug abuse with patient's tendency toward sedative drugs in Semnan prison. Asian J Med Pharm Res 2014;4:40-5.

5. McCabe SE. Correlates of nonmedical use of prescription benzodiazepine anxiolytics: results from a national survey of U.S. college students. Drug Alcohol Depend 2005;79:53-62.

6. Boyd CJ, McCabe SE. Coming to terms with the nonmedical use of prescription medications. Subst Abuse Treat Prev Policy 2008;3:22.

7. McLarnon ME, Monaghan TL, Stewart SH, Barrett SP. Drug misuse and diversion in adults prescribed anxiolytics and sedatives. Pharmacotherapy 2011;31:262-72.

8. Clark KD. The relationship of perceived stress and self-efficacy among correctional employees in close-security and medium-security-level institutions [dissertation]. Minneapolis (MN): Walden University; 2010.

9. Sadock BJ, Sadock VA. Kaplan and Sadock's comprehensive textbook of psychiatry. 8th ed. New York: Lippincott Williams and Wilkins; 2005.

10. Garcia-Montes JM, Zaldivar-Basurto F, Lopez-Rios F, Molina-Moreno A. The role of personality variables in drug abuse in a Spanish university population. Int J Ment Health Addict 2009;7:475-87.

11. Askari S, Zakiei A, Alikhani M. Personality characteristics and locus of control in relation to readiness for addiction among male university students. J Psychol Sci 2012;10:485-97.

12. Heshmaty R. Comparative study of alexithymia in patients with psychotic disorders, non-psychotic and normal subjects. Hamadan Med J 2010;7:59-68.

13. Lee YJ, Yu SH, Cho SJ, Cho IH, Koh SH, Kim SJ. Direct and indirect effects of the temperament and character on alexithymia: a pathway analysis with mood and anxiety. Compr Psychiatry 2010;51:201-6.

14. Taylor GJ, Bagby RM. New trends in alexithymia research. Psychother
Psychosom 2004;73:68-77.

15. Morrison SL, Pihl RO. Alexithymia and stimulus augmenting/reducing. J Clin Psychol 1990;46:730-6.

16. Evren C, Kose S, Sayar K, Ozcelik B, Borckardt JP, Elhai JD, et al. Alexithymia and temperament and character model of personality in alcohol-dependent Turkish men. Psychiatry Clin Neurosci 2008;62:371-8.

17. Loas G, Fremaux D, Otmani O, Lecercle C, Delahousse J. Is alexithymia a negative factor for maintaining abstinence?: a follow-up study. Compr Psychiatry 1997;38:296-9.

18. Sauvage L, Loas G. Criterion validity of Bermond-Vorst alexithymia questionnaire-20 form B: a study of 63 alcoholic subjects. Psychol Rep 2006;98:234-6.

19. Heidaripahlavan A, Mahjoob H, Rahimi A. Comparison of the prevalence of mental disorders in individuals addicted and non-addicted. Hamadan Med J 2011;18:22-8.

20. Hasanshahi M, Ahmadian K. Assessment of mental health in patients dependent on psychoactive substances. J Fundam Ment Health 2004; 6:131-9.

21. Karami J, Zakiei A, Mohebi Z. Relationship between alexithymia and beliefs about emotion with renal patient's mental health. Health Psychol 2013;4:19-29.

22. Noorbala AA, Mohammad K, Bagheri Yazdi SA. Validation of GHQ-28 in Iran. Hakim Res J 1999;5:101-10.

23. Picardi A, Abeni D, Melchi CF, Puddu P, Pasquini P. Psychiatric morbidity in dermatological outpatients: an issue to be recognized. Br J Dermatol 2000;143:983-91.

24. Parker JD, Taylor GJ, Bagby RM. The 20-Item Toronto Alexithymia Scale: III. Reliability and factorial validity in a community population. J Psychosom Res 2003;55:269-75.

25. Taylor GJ, Bagby RM, Parker JD. The 20-Item Toronto Alexithymia Scale: IV. Reliability and factorial validity in different languages and cultures. J Psychosom Res 2003;55:277-83.

26. Zakiei A. The relationship between alexithymia, Ellis irrational beliefs, positive and negative affection on psychiatric disorders among college students in Razi University of Kermanshah, Iran [unpublished master's thesis]. Kermansh: Razi University; 2012.

27. Ghorbani N, Bing MN, Watson PJ, Davison HK, Mack DA. Self-reported emotional intelligence: construct similarity and functional dissimilarity of higher-order processing in Iran and the United States. Int J Psychol 2002;37:297-308.

28. Pozzi G, Bacigalupi M, Tempesta E. Comorbidity of drug dependence and other mental disorders: a two-phase study of prevalence at outpatient treatment centres in Italy. Drug Alcohol Depend 1997;46:69-77.

29. Charney DA, Paraherakis AM, Negrete JC, Gill KJ. The impact of depression on the outcome of addictions treatment. J Subst Abuse Treat 1998;15:123-30.

30. Brown SA, Goldman MS, Inn A, Anderson LR. Expectations of reinforcement from alcohol: their domain and relation to drinking patterns. J Consult Clin Psychol 1980;48:419-26.

31. O'Boyle M, Brandon EA. Suicide attempts, substance abuse, and personality. J Subst Abuse Treat 1998;15:353-6. 